\title{
UM MODELO DE PASSO INVARIANTE BASEADO NA FUNÇÃO DE GOMPERTZ PARA PROGNOSE DO CRESCIMENTO ${ }^{1}$
}

\author{
DANIEL PEREIRA GUIMARÃES²
}

\begin{abstract}
RESUMO - Apresenta-se um modelo de passo invariante baseado na função de Gompertz. O ajuste do modelo foi efetuado em dados de crescimento de frangos de corte e em parcelas permanentes de plantações de Eucalyptus grandis. O modelo proposto apresenta a forma $\mathrm{Pf}=\mathrm{Pa} \cdot \exp \left(\beta_{0} \cdot \mathrm{S}^{\beta 2}\left(\exp \left(\beta_{1}\right.\right.\right.$.Ia $\left.)\right)-\left(\exp \left(\beta_{1}\right.\right.$.If $\left.\left.)\right)\right)$, em que Pf é a produção estimada na idade futura (If) a partir da produção atual $(\mathrm{Pa})$, observada na idade atual (Ia). S representa a introdução de nova variável ao modelo, sendo usada como variável binária representando machos e fêmeas no caso de frangos, e variável indicativa da qualidade do sítio no caso do eucalipto. As principais vantagens da utilização do modelo proposto residem na compatibilidade dos resultados, advinda da característica de passo invariante, projeções individuais e análise dos erros em diferentes intervalos temporais.
\end{abstract}

Termos para indexação: modelos não-lineares, frangos de corte, eucalipto.

\section{A STEP INVARIANT MODEL BASED ON GOMPERTZ'S FUNCTION FOR GROWTH PROGNOSIS}

\begin{abstract}
A step invariant model based on Gompertz's function is presented. Model adjustment was done on broilers and Eucalyptus grandis growth data. The proposed model presents the form $\mathrm{Pf}=\mathrm{Pa} \cdot \exp \left(\beta_{0} \cdot S^{\beta 2}\left(\exp \left(\beta_{1}\right.\right.\right.$.Ia) $)-\left(\exp \left(\beta_{1}\right.\right.$. If $\left.\left.)\right)\right)$, where $\mathrm{Pf}$ refers to the future estimated yield at age (If) based on yield (Pa) observed at present age (Ia). S means the introduction of a new variable to the model, being used as binarian variable to indicate male and female broilers and as a site quality for eucalypt. The main advantages of using the proposed model resides in the compatibility of results, assured by the step invariant characteristics, individuals projections and analysis of errors based on the prognosis intervals.
\end{abstract}

Index terms: nonlinear models, broilers, eucalypt.

\section{INTRODUÇÃO}

A explicação do crescimento biológico por meio de modelos contribui bastante para o desenvolvimento e teste de teorias a respeito do fenômeno. Conforme Titus \& Morton (1985), o processo de modelagem refere-se à busca de simplificações para explicar transformações de natureza complexa, sendo, portanto, apenas abstrações do sistema real.

\footnotetext{
${ }^{1}$ Aceito para publicação em 15 de abril de 1999.

2 Eng. Florestal, D.Sc., Embrapa-Centro de Pesquisa Agropecuária dos Cerrados (CPAC), Caixa Postal 08223, CEP 73301-970 Planaltina, DF. E-mail: daniel@cpac.embrapa.br
} 
Em termos práticos, as principais contribuições do uso de modelos referem-se a: identificação das principais variáveis associadas ao fenômeno em estudo, avaliação de mudanças decorrentes de alterações provocadas no processo estocástico (tratamentos), análise de tendências, o que permite o estabelecimento de projeções passadas e futuras, e fornecimento de subsídios para proceder análises econômicas e tomadas de decisão a priori.

De acordo com Pachepsky et al. (1996), o modelo mais eficiente será aquele que: possuir o menor número de parâmetros para uma determinada precisão das estimativas, representar a forma mais simplificada, estar em consonância com leis físicas, químicas ou biológicas, apresentar os menores desvios entre valores observados e preditos, e fornecer a menor variância dos estimadores.

Dentro desse contexto, grande ênfase tem sido dada ao emprego de modelos não-lineares, especialmente os que apresentam características assintóticas. Como base para o processo de modelagem, as funções Normal, Gama, Gompertz, Weibull, Sb Jonhson e Richards têm sido as mais empregadas. As principais vantagens de utilização dessas funções devem-se às características de descreverem curvas de distribuição nas formas derivadas e curvas sigmoidais nas formas integrais. Entre essas, a função Weibull tem-se destacado, graças às características de simplicidade e flexibilidade. Conforme Johnson \& Kotz (1970), a função de distribuição Weibull é atualmente a terceira função mais empregada em estatística, sendo suplantada apenas pelas distribuições Normal e Gama.

Um modelo de passo invariante, conforme Somers \& Farrar Junior (1991) apresenta a propriedade matemática em que predições sucessivas, efetuadas entre os passos $I_{1}$ e $I_{2}$ e de $I_{2}$ para $I_{3}$ apresentem os mesmos resultados obtidos pelo passo entre $I_{1}$ e $I_{3}$. Murphy (1983) estima a produção florestal por esse processo por meio da integração da função Richards. Guimarães (1994) avalia as mudanças nas distribuições diamétricas de Eucalyptus aplicando um modelo de passo invariante baseado na função Weibull.

Este trabalho apresenta um modelo de passo invariante baseado na função Gompertz. As aplicações do modelo são demonstradas pelo ajuste a dados relativos ao crescimento de frangos de corte e parcelas permanentes de um povoamento de Eucalyptus grandis. Sua escolha é justificada pelo fato de apresentar reduzido número de parâmetros e possuir curvatura similar à observada na tendência do crescimento de muitos seres vivos.

\section{METODOLOGIA}

\section{Desenvolvimento do modelo}

\footnotetext{
A função Gompertz pode ser expressa por: $\omega=A\left(\exp \left(-\exp \left(\beta_{0}+\beta_{1} \mathrm{t}\right)\right)\right)$ onde: $\omega$ representa a magnitude da variável dependente; $A$ corresponde ao valor assintótico; t é a magnitude da variável independente (normalmente o tempo); $\beta_{0}$ e $\beta_{1}$ são respectivamente os parâmetros determinantes da magnitude inicial $(t=0)$ e taxa de expansão. Essa função é classificada como de curvatura rígida, uma vez que apresenta ponto de inflexão constante e coincidente com o inverso da base dos logaritmos neperianos $(I=1 / \exp =0,37)$, implicando apresentar função de distribuição assimétrica à direita.
} 
A transformação dessa função em modelo de passo invariante resulta em: $P_{\mathrm{f}}=\mathrm{P}_{\mathrm{a}} \exp \left(\beta_{0}\left(\exp \left(\beta_{1} \mathrm{I}_{\mathrm{a}}\right)\right)-\left(\exp \left(\beta_{1} \mathrm{I}_{\mathrm{f}}\right)\right)\right)$

sendo $\mathrm{P}_{\mathrm{f}}$ a dimensão esperada na idade futura $\left(\mathrm{I}_{\mathrm{f}}\right)$ e $\mathrm{Pa}$, a dimensão observada na idade atual $\left(\mathrm{I}_{\mathrm{a}}\right)$.

$\beta_{0}$ ou $\beta_{1}$ podem ser substituídos por um vetor de parâmetros associados a novas variáveis, buscando-se obter um modelo com maior capacidade explicativa das tendências observadas.

\section{Aplicação do modelo}

O modelo foi aplicado a dados de crescimento de frangos de corte apresentados por Freitas et al. (1984) e a dados relativos ao crescimento volumétrico de Eucalyptus grandis, obtidos de 30 parcelas permanentes instaladas no Vale do Rio Doce, MG. Os dados referentes ao crescimento de Eucalyptus são mostrados na Tabela 1. Além da

TABELA 1. Produtividade de Eucalyptus grandis em função da idade e índice de sítio.

\begin{tabular}{|c|c|c|c|c|c|c|c|c|c|}
\hline \multirow[t]{2}{*}{ Parcela } & \multicolumn{8}{|c|}{ Idade de medição (meses) } & \multirow[t]{2}{*}{ Sítio } \\
\hline & 28 & 43 & 54 & 66 & 85 & 91 & 106 & 130 & \\
\hline \multicolumn{10}{|c|}{ Volume $\left(\mathrm{m}^{3} / \mathrm{ha}\right)$} \\
\hline 1 & 90,2 & 196,2 & 266,0 & 315,1 & 361,4 & 392,2 & 427,0 & 468,8 & 27 \\
\hline 2 & 14,6 & 41,6 & 56,1 & 73,2 & 93,8 & 106,1 & 118,3 & 133,4 & 18 \\
\hline 3 & 60,6 & 139,1 & 194,7 & 231,5 & 262,7 & 280,8 & 307,4 & 339,6 & 26 \\
\hline 4 & 86,7 & 189,7 & 260,0 & 302,0 & 360,6 & 377,7 & 411,4 & 452,0 & 28 \\
\hline 5 & 35,0 & 86,9 & 117,5 & 139,8 & 166,8 & 179,4 & 198,0 & 220,7 & 22 \\
\hline 6 & 21,2 & 56,9 & 88,4 & 119,2 & 157,5 & 172,2 & 190,2 & 212,2 & 20 \\
\hline 7 & 72,3 & 162,0 & 205,3 & 244,0 & 299,3 & 317,3 & 346,7 & 382,1 & 28 \\
\hline 8 & 32,5 & 81,7 & 119,9 & 146,9 & 181,5 & 198,4 & 218,6 & 243,2 & 20 \\
\hline 9 & 22,9 & 60,7 & 81,7 & 99,0 & 123,4 & 132,3 & 146,9 & 164,8 & 19 \\
\hline 10 & 56,1 & 130,2 & 216,1 & 278,6 & 341,4 & 370,7 & 403,9 & 443,9 & 28 \\
\hline 11 & 101,1 & 216,7 & 307,4 & 366,4 & 439,4 & 486,7 & 528,0 & 577,6 & 28 \\
\hline 12 & 95,3 & 205,8 & 283,4 & 349,3 & 408,2 & 444,1 & 482,5 & 528,6 & 27 \\
\hline 13 & 27,3 & 70,5 & 103,1 & 128,2 & 166,1 & 177,4 & 195,8 & 218,3 & 21 \\
\hline 14 & 55,8 & 129,5 & 199,0 & 249,9 & 317,3 & 345,1 & 376,5 & 414,3 & 22 \\
\hline 15 & 32,8 & 82,3 & 125,4 & 160,1 & 199,5 & 213,7 & 235,1 & 261,1 & 23 \\
\hline 16 & 62,6 & 143,1 & 187,3 & 234,2 & 268,3 & 298,4 & 326,4 & 360,1 & 25 \\
\hline 17 & 68,8 & 155,1 & 230,7 & 288,2 & 336,3 & 354,5 & 386,5 & 425,2 & 24 \\
\hline 18 & 54,3 & 126,6 & 199,1 & 259,2 & 336,9 & 361,9 & 394,5 & 433,8 & 22 \\
\hline 19 & 30,0 & 76,2 & 117,5 & 150,9 & 197,3 & 212,6 & 233,9 & 259,8 & 21 \\
\hline 20 & 58,8 & 135,4 & 177,3 & 221,3 & 266,3 & 284,2 & 311,1 & 343,6 & 23 \\
\hline 21 & 61,1 & 140,0 & 199,1 & 246,3 & 308,2 & 330,2 & 360,4 & 397,0 & 22 \\
\hline 22 & 59,8 & 137,6 & 186,2 & 234,4 & 275,5 & 309,4 & 338,1 & 372,9 & 22 \\
\hline 23 & 21,0 & 56,4 & 83,1 & 104,7 & 129,5 & 150,7 & 166,9 & 186,7 & 19 \\
\hline 24 & 11,1 & 33,2 & 54,6 & 70,4 & 87,8 & 96,3 & 107,7 & 121,7 & 16 \\
\hline 25 & 59,7 & 137,4 & 208,0 & 254,0 & 303,6 & 324,2 & 354,0 & 390,0 & 25 \\
\hline 26 & 33,0 & 82,6 & 117,3 & 143,2 & 177,9 & 201,2 & 221,6 & 246,4 & 19 \\
\hline 27 & 64,4 & 146,6 & 201,2 & 246,9 & 297,5 & 333,8 & 364,3 & 401,2 & 23 \\
\hline 28 & 57,4 & 132,8 & 201,1 & 243,8 & 283,3 & 337,4 & 368,2 & 405,4 & 22 \\
\hline 29 & 61,7 & 141,3 & 193,0 & 231,5 & 270,9 & 310,6 & 339,5 & 374,3 & 24 \\
\hline 30 & 52,4 & 122,6 & 174,0 & 199,0 & 233,3 & 264,8 & 290,1 & 320,9 & 25 \\
\hline
\end{tabular}


idade, foram incluídas: as variáveis sexo dos frangos de corte (utilizando-se uma variável binária, macho = 1 e fêmea = 2); e índice de sítio para Eucalyptus. $\mathrm{O}$ ajuste do modelo foi efetuado utilizando-se o programa SAS (Statistical Analysis System) e empregando o método DUD do procedimento NLIN, tendo como base o modelo expresso na seguinte forma:

$P_{f}=P_{a} \cdot \exp \left(\beta_{0} \cdot S^{\beta 2}\left(\exp \left(\beta_{1} \cdot I_{a}\right)\right)-\left(\exp \left(\beta_{1} \cdot I_{f}\right)\right)\right)$

sendo $\mathrm{S}$ correspondente à variável sexo no caso de frangos e a índice de sítio no caso de Eucalyptus grandis.

\section{RESULTADOS}

Em ambos os casos, o processo iterativo adotado pelo procedimento de ajuste não-linear apresentou convergência na estimativa de parâmetros que minimizaram as somas de quadrados dos resíduos, e todas as variáveis incluídas no modelo contribuíram significativamente $(\alpha=0,05)$ para explicar as mudanças nas taxas do crescimento ao longo do tempo. Os resultados obtidos referentes ao ajuste do modelo aos dados de frangos de corte são apresentados na Tabela 2, e os referentes a Eucalyptus grandis, na Tabela 3.

A Fig. 1 exemplifica a aplicação do modelo a partir dos pesos obtidos em frangos de corte na idade de 32 dias após o nascimento. A característica de passo invariante define a habilidade do modelo em efetuar projeções passadas e futuras, além de determinar valor igual ao peso observado em relação à idade inicial de projeção.

Para o caso de Eucalyptus grandis, efetuaram-se as estimativas futuras da produtividade $\left(\mathrm{m}^{3}\right.$ de madeira), independentemente da idade inicial de prognose. Os erros advindos dessas estimativas são apresentados na Fig. 2.

TABELA 2. Estimativa dos parâmetros referentes ao ajuste do modelo a dados de crescimento de frangos de corte.

\begin{tabular}{cclcc}
\hline Parâmetro & Estimativa & Erro padrão & \multicolumn{2}{c}{ Intervalo de confiança assintótico (95\%) } \\
\cline { 4 - 5 } & & assintótico & Inferior & Superior \\
\hline$\beta_{0}$ & 4,79969 & 0,00740 & 4,78512 & 4,81426 \\
$\beta_{1}$ & $-0,03349$ & 0,000156 & $-0,03380$ & $-0,03318$ \\
$\beta_{2}$ & $-0,09604$ & 0,00359 & $-0,10301$ & $-0,08898$ \\
\hline
\end{tabular}

TABELA 3. Estimativa dos parâmetros referentes ao ajuste do modelo a dados de crescimento de Eucalyptus grandis.

\begin{tabular}{crrrc}
\hline Parâmetro & Estimativa & \multirow{2}{*}{$\begin{array}{c}\text { Erro padrão } \\
\text { assintótico }\end{array}$} & Intervalo de confiança assintótico (95\%) \\
\cline { 3 - 5 } & & 2,71133 & Inferior & Superior \\
\hline$\beta_{0}$ & 30,67334 & 25,35146 & 35,99523 \\
$\beta_{1}$ & $-0,03666$ & 0,00032 & $-0,03730$ & $-0,03602$ \\
$\beta_{2}$ & $-0,56408$ & 0,02762 & $-0,61830$ & $-0,50987$ \\
\hline
\end{tabular}


Verifica-se que, independentemente da idade inicial de prognose, cerca de $83 \%$ das estimativas foram efetuadas dentro do limite de erros de $\pm 10 \%$.

A Fig. 3 ilustra a aplicação do modelo. No caso, estimativas da produção volumétrica aos 130 meses (cerca de 11 anos) são efetuadas a partir dos dados obtidos aos 43 meses (cerca de três anos e meio).

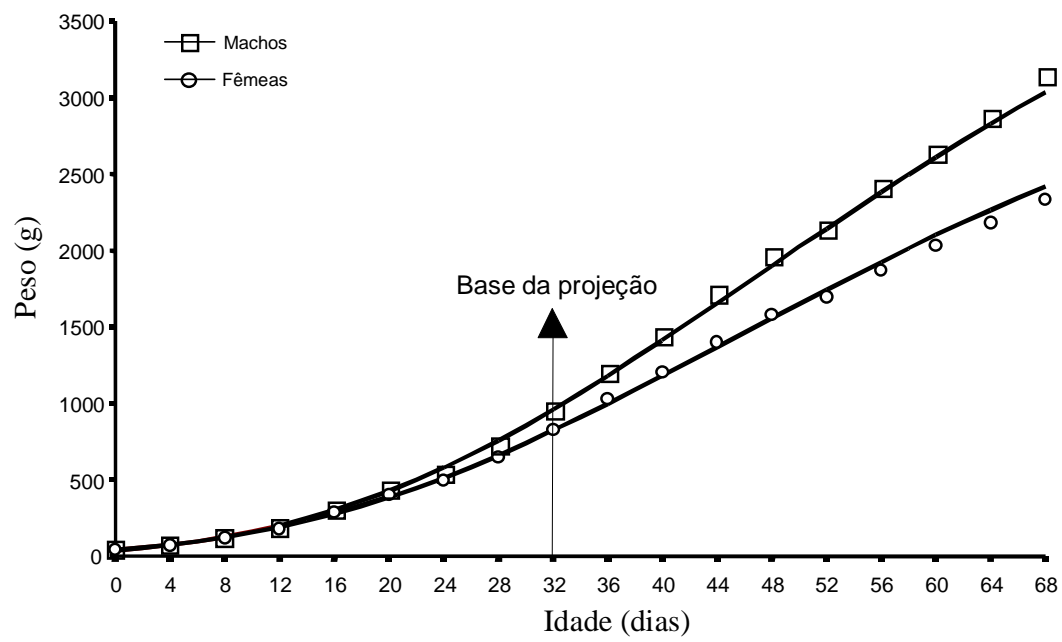

FIG. 1. Simulação do crescimento de frangos de corte a partir dos pesos corporais observados aos 32 dias.

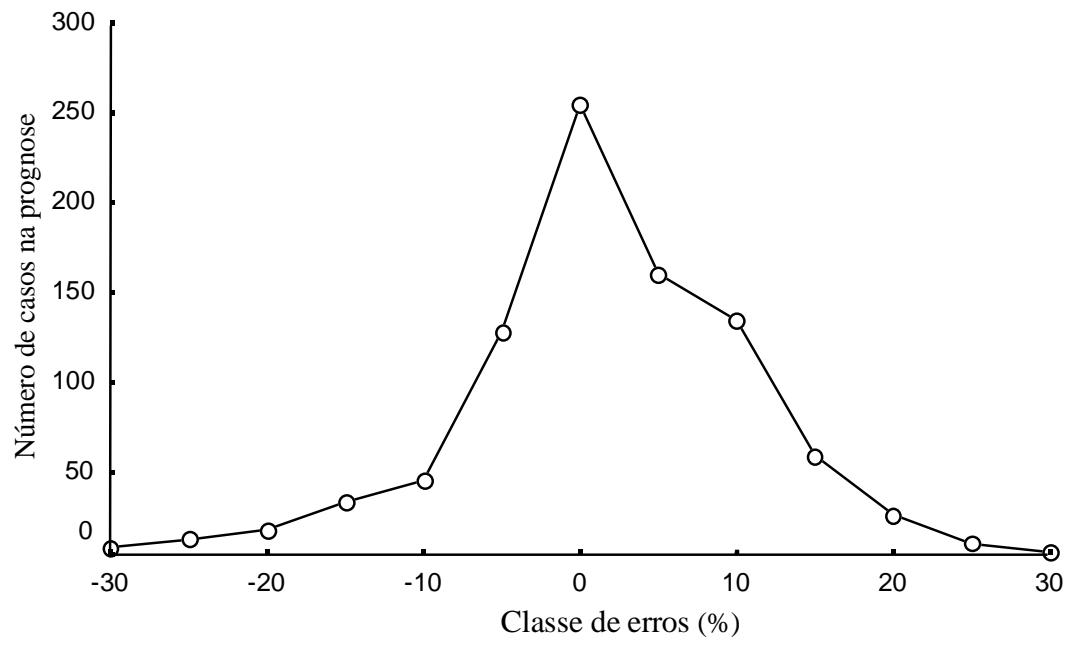

FIG. 2. Distribuição dos erros originários das estimativas das produções futuras de Eucalyptus grandis a partir do modelo de passo invariante. 


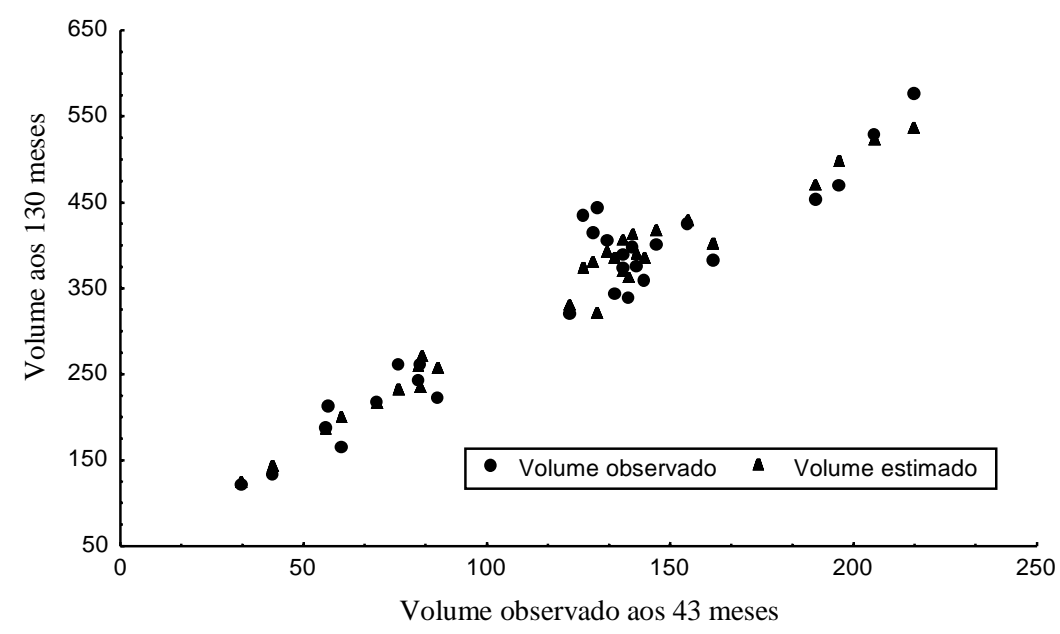

FIG. 3. Prognose da produção volumétrica de Eucalyptus grandis aos 130 meses a partir do volume e índice de sítio observados aos 43 meses de idade.

\section{CONCLUSÕES}

1. O modelo de passo invariante baseado na função Gompertz apresenta grandes possibilidades de uso na explicação do crescimento biológico de plantas e animais.

2. A utilização de um número reduzido de parâmetros (no caso, apenas três) e a forma simplificada são fatores que favorecem a seleção do modelo apresentado.

3. A transformação da função Gompertz em modelo de passo invariante oferece vantagens importantes no que se refere a: projeções individuais do crescimento, análise dos erros para quaisquer intervalos de projeção, o que permite definir a confiabilidade das estimativas em decorrência de diferentes intervalos temporais e estabelecer as idades iniciais a partir das quais o modelo deva ser empregado.

4. A introdução de novas variáveis ao modelo contribui para ampliar sua capacidade explicativa das tendências de crescimento sob o efeito de diferentes tratamentos.

5. A seleção das variáveis a serem empregadas constitui passo fundamental no processo de modelagem: no caso de frangos, variáveis associadas à alimentação (quantidade, teor de proteína na ração e outras) ou à disponibilidade de espaço (indivíduos/unidade de área), certamente seriam importantes; no caso de plantações florestais, torna-se possível o uso de variáveis associadas a parâmetros climáticos e edáficos. 


\section{REFERÊNCIAS}

FREITAS, A.R. de; ALBINO, L.F.T.; MICHELAN FILHO, T.; ROSSO, L.A. de. Modelos de curvas de crescimento em frangos de corte. Pesquisa Agropecuária Brasileira, Brasília, v.19, n.9, p.1057-1064, set. 1984.

GUIMARÃES, D.P. Desenvolvimento de um modelo de distribuição diamétrica de passo invariante para prognose e projeção da estrutura de povoamentos de eucalipto. Viçosa : UFV, Imprensa Universitária, 1994. 160p. Tese de Doutorado.

JOHNSON, N.L.; KOTZ, S. Distributions in statistics: continuous univariate distributions. Boston : Houghton Mifflin, 1970. 300p.

MURPHY, P.A. A nonlinear timber yield equation system for Loblolly Pine. Forest Science, Bethesda, v.29, n.3, p.582-591, 1983.

PACHEPSKY, L.B.; HASKETT, J.D.; ACOCK, B. An adequate model of photosynthesis. I. Parameterization, validation and comparison of models. Agricultural Systems, Essex, v.50, p.209-225, 1996.

SOMERS, G.L.; FARRAR JUNIOR, R.M. Biomathematical growth equations for natural longleaf pine stands. Forest Science, Bethesda, v.37, n.1, p.227-244, 1991.

TITUS, S.J.; MORTON, R.T. Forest stand models: what for? Forest Chronicle, Ottawa, v.61, n.1, p.19-22, 1985. 